\title{
Protée
}

\section{Poétique et sémiotique du titre musical}

\section{Claude Dauphin}

Volume 36, numéro 3, hiver 2008

Le titre des œuvres : accessoire, complément ou supplément

URI : https://id.erudit.org/iderudit/019630ar

DOI : https://doi.org/10.7202/019630ar

Aller au sommaire du numéro

\section{Éditeur(s)}

Département des arts et lettres - Université du Québec à Chicoutimi

\section{ISSN}

0300-3523 (imprimé)

1708-2307 (numérique)

Découvrir la revue

\section{Citer cet article}

Dauphin, C. (2008). Poétique et sémiotique du titre musical. Protée, 36(3), 11-22. https://doi.org/10.7202/019630ar

\section{Résumé de l'article}

Les indications de mouvements, allegro, adagio, presto, qui désignent les parties des sonates de Mozart, des concertos de Beethoven ou des symphonies de Brahms ont ceci de remarquable qu'elles pourraient aussi bien relever de l'abstraction la plus radicale que de la concrétisation la plus absolue. Ces intitulés expriment un état d'esprit, une disposition émotive, autant qu'ils énoncent la constitution de la musique. Je qualifie d'autonyme l'association du contenu et de la forme qui spécifie ces oeuvres et les procédés de leur intitulation. Mais la problématique des titres musicaux ne se résume pas à ce seul paradoxe car il existe bien, en contrepartie, une tradition du titre référentiel qui, à lui seul, semble se charger de toute la métaphore de l'oeuvre. De Couperin à Debussy, de Rameau à Messiaen, nous baignons dans une poétique de la caractérisation, de l'impression, du récit et de l'image : $L e$ Rossignol en amour, Les Langueurs tendres, Les Moissonneuses, L'Entretien des Muses, Les Féessont d'exquises danseuses, Couleurs de la Cité céleste. Au-delà de cette catégorisation, il apparait que le titre musical formel est relié à une esthétique résolument italo-allemande alors que la sensibilité française, plus visuelle qu'auditive, plus pittoresque qu'architecturale, privilégie l'intitulé référentiel. Ainsi, le titre ne se limiterait peut-être pas à la désignation de l'oeuvre musicale... Il dévoile la culture d'appartenance du compositeur et annonce une conception de l'art.
Ce document est protégé par la loi sur le droit d'auteur. L'utilisation des services d’Érudit (y compris la reproduction) est assujettie à sa politique d'utilisation que vous pouvez consulter en ligne.

https://apropos.erudit.org/fr/usagers/politique-dutilisation/ 


\section{POÉTIQUE ET SÉMIOTIQUE DU TITRE MUSICAL}

Claude Dauphin

On considère comme une certitude esthétique que l'œuvre musicale dite pure ${ }^{1}$, c'est-à-dire indépendante d'un texte poétique ou d'un livret, ne renvoie, en dernière analyse, qu'à elle-même. Michel de Chabanon, esthète subtil de la fin du XVIII siècle, formule admirablement ce topos autonymique de la musique qu'il a été probablement le premier à entrevoir: «Les sons [...] ne sont pas l'expression de la chose, ils sont la chose même» (1969: 168).

La certitude esthétique vacille pourtant lorsque certains textes littéraires, réels ou imaginaires, apposés à l'œuvre musicale, interfèrent avec l'axiome de l'autonymie musicale. C'est le cas lorsque la musique orchestrale accompagnée d'arguments narratifs appelés «programmes» se présente dans le genre de «symphonies à programme» ou de "poèmes symphoniques» qui a fait la fortune de maints compositeurs. Stravinsky lui-même, qui considérait «la musique, par son essence, impuissante à exprimer quoi que ce soit : un sentiment, une attitude, un état psychologique, un phénomène de nature, etc.» (2000: 69-70), a marqué l'histoire de cet art en donnant naissance aux œuvres orchestrales les plus évocatrices d'extériorité que l'on puisse imaginer. Arrimées au départ à des arguments de ballet, des œuvres comme L'Oiseau de feu (1910), Pétrouchka (1911) et Le Sacre du printemps (1913) mènent depuis une inépuisable carrière de musique d'orchestre dynamisée par le souvenir des contes populaires russes qui les ont inspirées. De tels récits se sont imprimés en filigrane de la texture orchestrale; ils orientent la réception de l'œuvre, forçant l'auditeur à décoder dans la musique entendue une histoire inscrite dans les sonorités instrumentales associées à des images, à des idées, voire à des intrigues et à des scénarios aussi authentiquement fantasmés que sont réelles les ondes qui affluent à nos tympans.

Est-ce à dire que, pour écouter aujourd'hui Le Sacre du printemps, nous devrions avoir l'argument du ballet en mémoire ou devant les yeux? Non! Le mélomane averti évite plutôt d'appeler la littérature au secours du musical; il abhorre d'être contraint à une translation apparentée à la synesthésie: une transcription littéraire qui rendrait intelligible ce que la musique chante. Les titres des parties de l'œuvre suffisent à guider l'évolution de l'action et à stimuler en deux temps et douze 
scènes dramatiques l'imagination de l'auditeur:

1. Le Baiser à la terre: a) "Augures printaniers",

b) "Danse des adolescentes", c) "Jeu du rapt»,

d) «Rondes printanières", e) «Jeu des cités rivales",

f) «Cortège du Sage», g) «Danse de la Terre»;

2. Le Grand Sacrifice: a) «Cercle mystérieux des adolescentes", b) «Glorification de l'élue», c) «Évocation des ancêtres», d) «Action rituelle des ancêtres», e) «Danse sacrale».

Cette efficace symbiose du syntagme littéraire et de la texture musicale n'est pas un artifice du $\mathrm{XX}^{\mathrm{e}}$ siècle: le programme de la Symphonie fantastique (1830); qui narrait les visions délirantes d'un jeune musicien noyé dans les vapeurs de l'opium, était non pas donné à l'auditeur mais publié dans les journaux les jours précédant le concert selon le vou de Berlioz. Le spectateur arrivait dans la salle tout "préparé» à décoder l'œuvre, à laisser voguer son imagination «chargée» sur les parties du drame musical. Le menu du "spectacle sonore», disons le "programme de la soirée», ne comportait que les titres, efficaces évocations des péripéties du héros:

1. «Rêveries et Passions»; 2. "Un Bal»; 3. "Scène aux champs»; 4. "Marche au supplice»; 5. "Songe d'une nuit de sabbat».

Ainsi se présente le titre d'une œuvre musicale: texte minimal, mot-clé, syntagme apposé en regard du discours sonore, visant à le décrire ou à le doter d'une charge narrative ou d'une intention allégorique. Dans cet article, je me propose de tracer les contours des différentes sortes de titres que l'on retrouve en tête des œuvres du répertoire instrumental. Malgré leur apparente infinité, ils se résument dans mon esprit à deux types: primo, ceux qui désignent l'allure expressive du morceau musical, son mouvement intérieur même et que j'appelle, en raison de cette intime allégorie du mot et de la chose, autonymes; secundo, ceux qui, renvoyant à une réalité extérieure, annoncent dans l'œuvre une métaphore sonore des faits évoqués. Je les nomme référentiels. Les titres autonymes aspirent à connoter dans le geste musical immanent des attitudes cinétiques observables physiquement ou intériorisées dans la conscience. Les titres référentiels inclinent à dénoter dans le continuum musical une poétique de faits, d'arguments, d'incidents, de caractères psychologiques même. Tous deux concourent au resserrement du sens, à l'accomplissement sémiotique du discours musical.

J'éviterai très volontairement d'inclure dans cette étude les genres mixtes, c'est-à-dire ceux où la musique est tissée avec un texte littéraire chanté ou déclamé: hymne, Lied, chanson, mélodie accompagnée, opéra, mélodrame. Dans ce genre d'œuvres, on comprendra que le titre étend à la musique une signification d'abord inscrite dans un texte rituel, poétique ou dramatique. Des titres d'opéra, on serait toujours en droit de préciser l'origine littéraire (Manon Lescault de Puccini, d'après le roman de l'abbé Prévost; Il Barbiere de Siviglia / Le Barbier de Séville, de Rossini, d'après la pièce de Beaumarchais). Ces affiliations de la musique au littéraire sont aussi évidentes dans le Lied allemand (Die Schöne Müllerin / La Belle Meunière, de Schubert, sur des poèmes de Wilhelm Müller) que dans la mélodie française (La Bonne Chanson, de Fauré, sur les poèmes de Paul Verlaine). Elles sont présentes d'emblée dans la messe, le requiem, l'oratorio, la passion, la cantate, le motet, le Te Deum, l'anthem, le magnificat, le psaume, l'hymne et l'ode, que ces sortes de musique sacrée, si prisées dans les concerts spirituels, conservent leurs titres génériques ou qu'elles s'adaptent aux intentions du compositeur ou à la dédicace de l'ouvrage.

Mais avant d'entrer dans une analyse des procédés propres à chacune des deux sortes d'intitulation des œuvres musicales, je m'arrêterai un moment à d'autres généralités. Toutes ces considérations préliminaires permettront, je l'espère, de concevoir combien l'intitulé de l'œuvre musicale appartient à un univers conceptuel différent du monde symbolique de la musique. L'intitulation revient à créer un complexe hétérogène vers lequel se tournent tous les espoirs du mélomane et même du musicien de voir s'accomplir le miracle de la révélation du sens indicible de la musique. 
FONCTIONS ET PROPRIÉTÉS DU TITRE MUSICAL: LE PARADOXE DE L'ÉPHÉMĖRE ET DE L'ÉTERNITÉ

La première fonction du titre serait d'exprimer une recherche de congruence entre les mots du langage et le flux inarticulé 2 de la musique visant ainsi à conférer au discours musical une dimension poétique et «largement» significative, apte à combler l'absence de référent. L'adverbe italien "Adagio», signifiant «aisément, à une allure modérée», n'aurait que ce sens restreint dans le langage courant, sens qui pourrait être nuancé par le contexte de son emploi. Mais, en musique, l'état de grâce évoqué par ce seul mot prend valeur de méditation sonore, révélatrice d'une sérénité ponctuée par le rappel des tourments qui en ont motivé l'aspiration, et du bonheur éprouvé à son accomplissement. En fait, il n'y a rien d'explicite dans le tissu sonore de la page pour orchestre à cordes de Samuel Barber qui porte ce seul mot pour titre, sinon des pensées qui, selon le mot de Mendelssohn, «ne sont pas trop vagues [...] pour être saisies par la parole, mais au contraire, trop précises pour cela ${ }^{3}$ : tout un univers d'indicibles.

L'intitulation d'une œuvre consisterait, en second lieu, à lui assigner une identité symbolique permettant de la reconnaître, de l'appeler, de la désigner parmi la myriade d'artéfacts dont les civilisations humaines sont parsemées. Le titre assigne à l'œuvre une place dans le catalogue patrimonial de l'humanité en la dotant d'identité et de pérennité. Cette matérialisation symbolique exercée par le titre s'avère d'autant plus prégnante que les propriétés perceptibles de l'objet désigné sont évanescentes. Ce paradoxe s'exerce tout particulièrement sur les arts de la scène. Pour la danse, le théâtre et la musique, le temps représente le paramètre essentiel de leur actualisation. Ces formes de production aspirent à l'éternité en dépit de leur incarnation éphémère qui coïncide avec le temps de la représentation de l'œuvre. Qu'importe que l'œuvre soit condamnée au mutisme si l'icône de son seul nom permet de l'évoquer encore. La fonction du titre réside en cette "capacité de représenter l'absent» qui, selon Jean Paulus, serait le principal attribut du symbole (1972: 21).
Privée de l'incontournable dimension spatiale qui tempère la tyrannie du temps comme au théâtre et en danse, la musique semble livrée à l'éphémère. Confrontée à sa fugitive temporalité, elle conduit à une vertigineuse réflexion ontologique qui se cristallise, entre autres, dans l'intitulation de l'œuvre. S'il peut être difficile de définir la matérialité de l'œuvre scénique manifestée sous différentes formes un manuscrit d'auteur, sa reproduction, son contenu, sa mise en scène, son interprétation-, l'équivoque s'amplifie dans le cas de la musique où la pièce peut être esquissée, interprétée par tout un chacun sur un instrument qu'il maîtrise peu ou prou, en chantant, en fredonnant ou en sifflotant tout ce que sa mémoire lui permettrait d'en restituer. Laquelle de ces exécutions détient le certificat d'authenticité d'un morceau de musique? À laquelle pourrait-on le contester? En effet, comme l'a si bien démontré Roman Ingarten:

L'ouvre musicale, en tant qu'objet de la perception esthétique, n'est pas en elle-même un événement réel ou un objet réel. Selon son contenu qui seul devient visible au moment de la perception esthétique, elle ne se relie causalement à aucun processus, à aucun fait réel. Par contre, chaque exécution de l'œuvre musicale est réelle. (1989: 80-81)

À l'objection de l'appartenance de l'œuvre à une culture, à une époque, à une société, aux considérations sociologiques qui la font ranger parmi les produits et les faits générés "par la trame de la vie d'une époque historique» (ibid.: 81), Ingarten réplique:

Tout ce qui est créé dans un temps donné ne doit pas nécessairement, par ce fait, être quelque chose de réel. L'arcen-ciel, par exemple, provient de causes réelles dans un temps déterminé, mais en soi, n'est rien de réel. (Ibid.)

La métaphore est saisissante: la brièveté et l'impalpabilité de l'œuvre musicale la mettent dans la situation d'un phénomène purement illusoire. Tout art consiste bien sûr à créer l'illusion, mais cette création s'incarne dans des masses, s'implante dans un espace, se matérialise dans l'illusion même de défier le 
temps et non se fondre en lui comme le fait l'art des sons.

Face à l'inévitable domination du temps sur les êtres, les choses et les phénomènes, demeure le mince espoir de leur appellation, car il s'avère que cette symbolisation prolonge la mémoire d'une existence même après son évanouissement. Le titre permettrait ainsi de conjurer la fuyante temporalité qui condamne la musique à une abstraite matérialité sonore et la fait perpétuellement osciller entre incarnation et volatilité à l'image même du message plurivalent qu'elle délivre. Le dernier recours de la musique à l'existence consisterait justement à sonoriser l'écoulement du temps en créant des repères événementiels faits de motifs rythmiques, de thèmes mélodiques, de zones de timbres et d'ambiances harmoniques que nous appelons «l'œuvre».

Ces enjeux ontologiques se traduisent bien prosaïquement dans la fonction classificatoire perceptible au premier plan de l'intitulé musical. La préoccupation taxinomique décline ces termes désignatifs si courants dans les intitulés musicaux: terme générique (symphonie ou sonate), terme ordinal dans la chronologie des productions du compositeur (opus), terme ordinal dans un recueil comportant plusieurs œuvres (numéro), à quoi l'on ajoute encore la tonalité et les instruments auxquels l'œuvre est destinée. L'on est en présence d'une arborisation. Ainsi, le Concerto dit «Empereur» de Beethoven ne sera correctement désigné qu'avec la mention de ses entités concertantes, "pour piano et orchestre", suivie de son numéro ordinal parmi les cinq œuvres de cette espèce retenues au catalogue du compositeur, "numéro 5 ", à quoi il convient encore d'ajouter la tonalité principale des mouvements extrêmes, "en mi bémol majeur», sans oublier le numéro de l'œuvre au catalogue, «opus 73 ». Il est à remarquer que le compositeur récusait énergiquement cette épithète impériale apocryphe attribuée à son dernier concerto de piano. Mais du public, de la critique et des éditeurs, les voix se sont élevées pour encourager cette nouvelle surenchère et en affubler une appellation déjà surchargée.
Comment justifier cette ramification désignative sinon par le caractère «impalpable» de l'œuvre sonore? En comparaison, l'intitulé d'une peinture contemporaine du Concerto Empereur (1809-1811) ne serait point pourvu d'autant de paramètres désignatifs. La Grande Odalisque (1814) d'Ingres n'a besoin d'autre parure que son titre pour la désigner. Si d'aventure d'autres informations accompagnaient l'intitulé, ce serait pour indiquer le musée qui possède le tableau, le Louvre en l'occurrence, et sa localisation à Paris afin d'orienter le public vers la femme nue négligemment allongée sur son récamier. Cette matérialité de l'œuvre picturale originale assure sa pérennité et clame sa réelle présence en un lieu donné. Les amateurs vont la voir pour le plaisir de rêver, comme les auditeurs s'empresseront d'aller écouter l'Empereur de Beethoven, une œuvre en revanche dotée d'ubiquité, puisque pouvant être exécutée simultanément à Montréal, à New York ou à Chicago par des orchestres concurrents dirigés par des chefs rivaux qui ne se seraient point consultés. Il importe que des symboles iconiques forts assurent un certain contour à ces mouvantes conformités sonores dont la consignation originale, le manuscrit autographe conservé à la bibliothèque d'État de Berlin, n'a probablement jamais été vue par les musiciens qui l'exécutent.

\section{REFLETS DE L'AUTONYMIE MUSICALE DANS L'ARBRE DU TITRE \\ Tel se présente le titre musical dans sa morphologie arborescente qui ne se limite point à l'énonciation du genre: suite, partita, toccata, sonate, symphonie ou concerto. Les titres de cette sorte ont été fréquemment associés aux œuvres instrumentales pures, c'est-à-dire dénuées de prétention picturale ou programmatique. Très prisées dans les périodes baroque, classique et romantique caractérisées justement par la profusion des compositeurs et l'abondance des œuvres, ces dénominations génériques peuvent effectivement sembler dépourvues d'imagination poétique et ne recéler que des indices formels gommant toute préoccupation expressive.}


En réalité, elles participent de la congruence entre syntagme littéraire et discours musical. Elles sont à l'expression musicale ce que la figure est au sublime, comme l'exprimait le pseudo-Longin ( $\mathrm{I}^{\mathrm{er}} \mathrm{ou}$ III $\mathrm{e}$ siècle): «si les Figures soutiennent naturellement le Sublime, le Sublime de son côté soutient merveilleusement les Figures" (1995: 103).

Les rares auteurs qui se sont penchés sur la définition des intitulés de l'œuvre musicale ont eu tendance à dénigrer la pratique des titres «génériques». Telle est en tout cas la position de Françoise Escal qui marginalise visiblement la valeur de ces appellations «d'époque ancienne» auxquelles elle dénie toute "valence poétique» (1996: 192). Elle dénonce leur "pauvreté lexicale» et constate qu'il est

[...] difficile de parler de rhétorique ou de poétique des titres baroques et classiques: ils sont brefs, ne comportent ni chiasme, ni antithèse, ni oxymore ou paronomase. Un seul mot suffit en général, et c'est un substantif. (Ibid.: 204)

Escal parvient à une conclusion aussi dépréciative parce qu'elle ne considère que le paramètre générique des titres arborescents. Y font suite pourtant des compléments autrement significatifs qui désignent justement l'espace chiasmatique et antithétique du discours musical lui-même. Une œuvre relevant du genre "concerto" génère des espèces du type "grosso», «ripieno» ou «solo», qui se découpent en des moments d'allure spécifique, alternatifs et contrastants, sous-titrés Allegro, Largo, Presto ou des variantes nuancées de ces indications cinétiques. Il importe de compléter la nomenclature initiale en regardant au-delà de la prime mention du genre pour comprendre comment ces titres étagés épousent la poétique de la composition sonore. Cela rappelle les taxinomies botaniques: Iris cristata «Alba» et Iris germanica "Florentina». La désignation du genre est suivie de l'espèce et, dans ce dernier groupe, on nomme les individus. Il s'agit bien là d'une poétique formaliste de l'intitulation particulièrement adaptée au médium musical: une pratique descriptive, taxinomique, je le concède, mais combien révélatrice des prérogatives autonymiques de la musique.
Si l'art du titre autonymique comporte un enjeu ordinal, il faut d'abord comprendre qu'il s'agit là d'une survivance symbolique visant à désigner le principe premier de musique. En effet, et malgré les nuances qu'impose la distinction entre la mousikê de l'Antiquité et les conceptions de la musique dans la civilisation occidentale «moderne», on ne peut oublier que les théoriciens grecs définissaient la mousikê comme mise en ordre du monde, à telle enseigne que le mot lui-même pouvait être synonyme d'encyclopédie. Toute pratique de la musique semble porter en son essence les stigmates de cette taxinomie matricielle. Stravinsky s'accroche d'ailleurs à ce principe premier en stipulant que «le phénomène de la musique nous est donné à la seule fin d'instituer un ordre dans les choses, y compris surtout un ordre entre l'homme et le temps» (2000: 72). Le titre ne serait que l'insigne du rituel d'accomplissement de l'ordo mundi dans le discours musical.

Conséquemment, l'autonymie de la musique reflétée dans l'intitulé énonce une passion formaliste qui constitue un second niveau du rapport des compositeurs aux ouvres. Escal acclame la musique contemporaine parce qu'elle

[...] se déclare jeu de formes, et les titres alors essaient de décrire et d'expliquer des processus compositionnels, ils ne signifient plus le sujet de l'œeurre mais l'œeuvre comme objet.

J'abonde dans le même sens avec la réserve toutefois que cette "objectivation" reconnue dans l'intitulation des œuvres nouvelles ne s'oppose pas à la tradition d'autonymie mais, bien au contraire, la poursuit. C'est en considérant leurs œuvres comme objets que les compositeurs italiens et allemands des XVII et XVIII e siècles manifestaient à leur égard la passion du classement de leurs formes par l'annonce de leurs genres en déclinant les espèces de leurs subdivisions. Ces énoncés préparaient l'auditeur à se projeter symboliquement dans l'individuation du mouvement musical. Dans leurs pratiques d'intitulation autonymes, les compositeurs du XXe siècle s'appuient sur des représentations symboliques 
tout à fait semblables à celles de leurs devanciers. Les formules de désignation formelles se sont simplement diversifiées avec la multiplication des formes résultant d'associations plus serrées avec d'autres sphères de la représentation symbolique, comme les mathématiques et la géométrie. Comme Escal en convient d'ailleurs, Olivier Messiaen (1908-1992), en intitulant l'une de ses œuvres Modes de valeurs et d'intensité, ne procède pas autrement que le Bach du Das Welltempierte Clavier / Le Clavier bien tempéré ou du Die Kunst der Fugue / L'Art de la fugue. Ces vocables signalent une même intention révélatrice de l'élaboration formelle et technique de l'œuvre.

\section{L'INTITULATION RÉFÉRENTIELLE:}

\section{UNE POÉTIQUE FRANÇAISE DE LA MUSIQUE}

Force est de constater cependant que la désignation de l'œuvre dans son genre, sa forme et ses modalités expressives intrinsèques relève d'une pratique prédominante dans les traditions italiennes et allemandes, du moins jusqu'au XIXe siècle. De leur côté, les compositeurs français, de la Renaissance à nos jours, privilégient l'intitulation relative à des sujets extérieurs à l'œuvre. La conception française de l'intitulé semble à la limite représenter une forme de déni de l'autonymie musicale. Elle instaure un parti pris référentialiste qui rapproche la musique du figuralisme en peinture. Elle invite l'auditeur, à tout le moins, à associer le discours musical à des référents extérieurs audibles, visuels, voire narratifs. Cette recherche française de la caractérisation ouvre un tout autre horizon à la poétique musicale. Son esthétique transcende les époques puisqu'on la retrouve intacte dans la célèbre "fantaisie zoologique» de Camille SaintSaëns, Le Carnaval des animaux (1886), chez Darius Milhaud, «La Création du monde» (1919), «Le Bouf sur le toit» (1923) et chez Messiaen, "Des canyons aux étoiles» (1974). Elle ne s'est bien sûr pas limitée à la France, car certains compositeurs allemands - Telemann, Haydn et Strauss ${ }^{4}$ - ou italiens - Vivaldi, Respighi ${ }^{5}$ - ont cédé à son attrait. En outre, sa poétique est partagée par d'autres nations comme l'Angleterre ${ }^{6}$ ou la Russie 7 . Mais son épicentre demeure la France.
Le privilège accordé par les Français à l'allusion externe s'impose déjà à la Renaissance. Les compositeurs d'alors empruntaient les titres fleuris de leurs chansons polyphoniques aux premiers vers des poèmes de Du Bellay, de Baif et de Ronsard. Du «Prince des poètes» de La Pléiade, Anthoine de Bertrand (1510-1577) a mis en musique Les Amours de Cassandre où les titres des sonnets sont plus évocateurs les uns que les autres: "Je parangonne au Soleil que j'adore", "Ce ris plus doux que l'œuvre d'une abeille», "Certes mon œil fut trop aventureux». Mais sitôt que la pièce se prêtait à une parodie instrumentale, comme l'habitude s'affirmait de plus en plus avec l'essor de la lutherie, l'allusion au poème était supplantée par des titres qui n'avaient de la désignation générique que l'apparence. L'incipit des trois sonnets de Ronsard tout juste cités aurait pu donner prétexte à des compositions, pour le luth ou pour la viole de gambe, intitulées pavane, fantaisie ou gaillarde. Loin de se limiter à désigner des genres musicaux, chacune de ces appellations entraîne dans son sillage d'autres valeurs sémantiques: pavane, c'est-à-dire avec fierté, arrogance, en faisant étalage de sa parure à la manière du paon; fantaisie, c'est-à-dire à l'improviste, selon l'humeur ou le goût du moment; gaillarde, c'est-à-dire de façon vive, osée, à la limite licencieuse. Ce faisant, ces trois génériques connotent aussi bien des gestes et des attitudes physiques cristallisés dans la danse que des conduites morales ou des états d'âme que la musique prétend caractériser. Ainsi l'intitulation française fait-elle converger deux faisceaux de significations - l'une physique, l'autre morale - qui soutiennent un argument-récit stylisé dans la représentation musicale.

C'est dans ce cadre esthétique fondé sur une poétique référentielle que s'inscrit l'expérience musicale française. Cette dernière se modélise par la figure et le récit. Alors, le discours musical incline à s'associer des faits situés au-delà du tissu sonore perceptible. Sa conjugaison avec la nomenclature des danses et avec les syntagmes littéraires devient alors inéluctable. L'étude de l'intitulation des pièces du répertoire français prend, dans cette perspective, une valeur exemplaire. 


\section{LA DANSE COMME FIGURE}

\section{DE L'INTITULÉ RÉFÉRENTIEL}

Quelle place occupe la nomenclature chorégraphique dans ce complexe d'adéquations instaurées par l'intitulation référentielle et le discours musical immanent? La suite représente le genre originel où s'organise l'opération référentielle activée par l'intitulé des morceaux qui la constituent. Elle est d'abord reconnaissable par le nombre de ces derniers, habituellement supérieur aux trois mouvements réglementaires qui façonnent la sonate ou les quatre qui modèlent la symphonie. En outre, tout au long de la période baroque (XVII et XVIII e siècles), les parties de la suite, précédées d'un Prélude, rapportées à une tonalité unique ou à des tonalités adjonctives, étaient désignées par des titres de danses: Allemande, Courante, Sarabande, Menuet, Rondeau, Gigue. L'Allemande, à quatre temps, lente et bien affirmée, n'est pas une danse allemande comme son titre pourrait le laisser croire. Cette désignation traduit plutôt une perception française de la nation voisine d'outreRhin, car la danse paysanne réellement allemande, le Ländler, à trois temps, plutôt légère, anticipe la valse. La Courante symbolise l'Italie d'où on la croit issue. Sa métrique ternaire composée la prédispose à être prompte et fluide, courante, comme le dit son nom; en quoi elle s'apparente aux genres de la musique méridionale comme la sicilienne et la tarentelle. La Sarabande, à trois temps marqués, progresse lentement, grave, imposante et orgueilleuse, avec ses motifs en arabesque dévoilant ses origines hispano-mauresques. Le Menuet, à trois temps fluides, subtil et élégant, suggère les choses délicates et les personnes menues. Il pastiche les allures des nobles. Le Rondeau, binaire, tournoie, naïf et léger, comme une ronde d'enfants. La Gigue, binaire, sautille, capricieuse et frondeuse comme le sont ces capricornes pourvus de gigots. Elle symbolise les peuples d'outre-Manche.

Le spectacle intérieur auquel convie la suite instrumentale est celui du caractère des nations et, à l'intérieur de ces dernières, de l'affectation des aristocrates et du naturel des paysans. Image des peuples et de leur hiérarchie sociale, la suite instrumentale corrobore une passion ethnologique et sociologique proprement française aux XVII et XVIII siècles, comme en témoignent ces thématiques dans les œuvres littéraires de La Bruyère (Les Caractères), de Montesquieu (L'Esprit des Lois, Les Lettres persanes), de Voltaire (Zadig, Candide, L'Ingénu). Genre éminemment français, la suite fournit aux compositeurs des autres nations un champ d'exercice compositionnel très valorisé en une époque où la reconnaissance du métier requiert une maîtrise de trois grands styles nationaux: italien, allemand et français. Depuis la fin du XVI e siècle, les compositeurs italiens s'ingéniaient à réaliser des versions instrumentales des canzone francese en caractérisant les attitudes physiques et les comportements moraux musicalisés par des intitulés chorégraphiques. Pour eux comme pour les Allemands, la caractérisation représentait déjà une typologie française. C'est dans leur sillage qu'Arcangelo Corelli (1653-1713) et Johann-Sebastian Bach (1685-1750) ont pu asseoir leur réputation de musiciens européens en démontrant par leurs suites de danses qu'ils maîtrisaient l'art français de la caractérisation musicale 8 aussi bien que l'univers clos de la sonate et de la toccate.

\section{LE PORTRAIT COMME CARACTÉRISATION MORALE:}

\section{LA FIGURE DE COUPERIN «LE GRAND»}

Si le titre référentiel, inductif de l'œuvre, puise son origine dans une pratique bien française dessinée depuis le XVI e siècle, il prend un tournant décisif et autrement affirmé avec la manière éblouissante dont François Couperin II (1668-1733), dit «le Grand», en a usé. Pour mettre en relief cette attention portée à l'intitulation référentielle généralisée dans la musique française depuis Couperin, le musicologue Pierre Saby utilise cette expression on ne peut plus pertinente: «l'artisanat du titre» (2008). Couperin justifie cette désignation par ses propos dans la préface du premier de ses quatre livres de Pièces de clavecin; il y dévoile son ambitieux projet de faire de ses suites une galerie de portraits:

J'ai toujours eu un objet en composant toutes ces pièces: des occasions différentes me l'ont fourni. Ainsi les Titres répondent 
aux idées que j'ay eues; on me dispensera d'en rendre compte; cependant [...] il est bon d'avertir que les pièces qui les portent sont des espéces de portraits qu'on a trouvé quelques fois assés ressemblans sous mes doigts, et que la plûpart de ces Titres avantageux sont plûtôt donnés aux aimables originaux que j'ay voulu representer, qu'aux copies que j'en ai tirées.

$(1980: 10)$

Il ressort de l'attentive observation accordée à son entourage quatre Livres de Pièces de clavecin (1713-1730) comportant vingt-sept cycles de tableaux sonores où chaque intitulé épingle une intention postulée par le compositeur pour sa figuration musicale. Couperin emploie le mot ordre pour désigner ses suites aux dimensions très fantaisistes. En réalité, chacun de ces ordres commence par décliner les attributs ordinaires de la suite pour s'en éloigner ensuite. Les trois premiers ordres annoncent la manière en caractérisant l'appellation convenue de plusieurs des danses par un titre adjoint. Ainsi, l'Allemande sera tantôt "Auguste», "Laborieuse", "Ténébreuse»; la Sarabande, «Majestueuse», «Prude», «Lugubre». C'est alors, après avoir établi ces correspondances entre l'appel de danse et les caractères qui lui sont associés, que Couperin démultiplie les mouvements de l'ordre dont le nombre dépasse la vingtaine dans le cas des deux premières suites. Une fois débarrassé du cadre conventionnel de la suite par le fait de cette démultiplication des mouvements, le compositeur se libère aussi de la convention d'appellation chorégraphique: n'étant plus apparentés que par leurs tonalités, les mouvements sont traités comme autant de petits camaïeux où le sujet est désigné par des «titres-personnage» ${ }^{9}$, des "titres-caractère» ou des «titres-paysage »: «Les Nonètes (Blondes et Brunes)", «La Bourbonnoise», «La Manon», «L'Enchanteresse», «Les Abeilles», "La Nanète», «La Florentine», «La Garnier», «La Babet», «La Diligente», «La Flateuse», "La Voluptueuse», "Les Pèlerines», "La Pateline », «L'Espagnolète» «Les Matelotes Provençales», «La Favorite», «La Lutine», etc.

Certains intitulés s'éloignent du projet de portraiture pour esquisser une intrigue car la construction d'une chaine de correspondances linéaires pourvoit la suite française d'une capacité narrative inscrite dans la logique des intitulés. Ainsi chez Couperin, «Les Pèlerines» du Premier Livre (3e Ordre) donnent à suivre un récit évolutif en trois tableaux: «La Marche», «La Caristade», «Le Remerciement». Un argument implicite semble soutenir cette saynète musicale. Tableau 1 : les pèlerines marchent «gayement» vers le but de leur célébration. Tableau 2: durant leur épuisant périple, elles sollicitent "tendrement» la charité des habitants du pays qu'elles traversent. Tableau 3: elles expriment à leurs donateurs leur reconnaissance pour les faveurs reçues en dansant «légérement» comme des anges. Les trois moments des Bacchanales (4e Ordre) - «Enjouemens bachiques», «Tendresses bachiques» et «Fureurs bachiques" - dessinent aussi un petit drame conjugal autour du thème de l'ivresse. Il est à se demander parfois si la rime de certains titres ne suggère pas des correspondances entre les pièces d'un ordre à l'autre. Ainsi, «Les Idées heureuses» (Premier Livre, 2e Ordre) semblent annoncer quelque aventure galante bien engagée qui se buterait plus loin à quelque obstacle imprévu et inexplicable, «Les Barricades mystérieuses» (6e Ordre). L'esthétique musicale française issue de la suite se caractérise ainsi par l'impératif évolutif du récit: elle trouvera son aboutissement dans le poème symphonique de Berlioz (1803-1869), La Symphonie fantastique, de Paul Dukas (1865-1935), L'Apprenti sorcier, et de Camille SaintSaëns (1835-1921), la Danse macabre.

Le titre révèle la thématique de la pièce qui sans lui resterait totalement inaccessible. Il donne la clé de la signification de l'œuvre en dévoilant son objet de référence. Il articule le "hiéroglyphe musical» 10 au but de son analogie. Les morceaux prétendent portraiturer des personnes - «La Bersan», "La Tendre Fanchon», "La Princesse de Sens», «Le Gaillard boiteux» -, représenter des professions - «Les Calotins et les Calotines" -, peindre des scènes bucoliques - «Le Rossignol en amour», «Les Abeilles», «Les Moissonneurs» -, évoquer des péripéties - «Désordre et déroute de toute la troupe, causés par les Yvrognes, 
les Singes et les Ours». Il en est ainsi des 245 titres de mouvements ou de sections qui composent cet imposant monument de la littérature pour clavier que sont ces Pièces de clavecin du "Grand" Couperin.

L'esthétique de la caractérisation des pièces pour clavecin imposée par Couperin est infinie. Adoptée par ses contemporains Dagincourt (1684-1758) et Dandrieu ${ }^{11}$ (1682-1738), elle se prolonge sans peine dans les deux derniers recueils de Pièces de clavecin et dans les Cinq pièces pour clavecin seul extraites des Pièces pour clavecin en concerts de Jean-Philippe Rameau (1683-1764). Déjà dans son Premier Livre (1706), antérieur de sept ans au Premier Livre (1713) de Couperin, où il s'adonnait au genre de la suite de danses stylisées, Rameau introduisait une chantante et inhabituelle "Vénitienne» (barcarolle) au numéro 8. Le Deuxième Livre (1724) contient deux suites. La première en $\mathrm{mi}$ (majeur/mineur), aux appellations usuelles de danses, joue encore d'originalité avec un bucolique «Rappel des oiseaux» au numéro 5 , seule pièce descriptive de cette suite si l'on considère «La Villageoise» comme une épithète du «Rondeau» ordinaire et le "Tambourin» comme un qualificatif $\mathrm{du}$ «Rondeau» à la provençale. Mais la seconde suite, celle en ré (majeur/mineur), n'aligne que des titres référentiels: «titres-personnage» - «Les Niais de Sologne» -, «titres-caractère» - «La Joyeuse», «La Boiteuse» -, titres allégoriques - «L'Entretien des Muses» et «titres-paysage» - «Les Tourbillons». Après une première suite en la (majeur/mineur) où les danses sont encore de convention, à l'exception de deux morceaux sur lesquels je reviendrai, «Les Trois mains» et la "Fanfarinette», le Troisième Livre offre en deuxième partie de programme une suite en sol (majeur/mineur) ponctuée d'intitulations référentielles: « Les Tricotets», «L'Indifférente», «La Poule», «Les Sauvages», «L'Égyptienne». Je garde, ici encore, deux mouvements en vue d'un commentaire différé: «Les Triolets» et "L'Enharmonique». Enfin, toujours dans le registre de la référence extramusicale, les quatre portraits extraits des Cinq pièces pour clavecin seul ne peuvent être laissés pour compte: "La Livri», «gracieuse» effigie pour honorer la mémoire du comte de Livri, protecteur du compositeur, «L'Agaçante», «La Timide» et «L'Indiscrète».

\section{PAR DELÀ «LE TOMBEAU DE COUPERIN » 12}

L'intitulé des œuvres d'art, à quelque domaine qu'elles appartiennent, demeure un attribut de l'inspiration de l'artiste. Il s'agit d'un procédé foncièrement évolutif, nourri au terreau de l'imaginaire et auquel il serait bien présomptueux de prétendre fixer des limites. Aussi, le défi d'une étude de l'intitulation du musical ne saurait envisager l'exhaustivité des pratiques. Il m'est apparu néanmoins nécessaire de définir les typologies les plus fonctionnelles et les plus englobantes du titre musical, c'est-à-dire celles qui rendent compte des tendances cardinales de l'intitulation en accord avec les stratégies expressives généralement en œuvre dans la composition musicale. Toute tentative de souscatégorisation initierait un dédale de subdivisions faussement prometteuses d'exhaustivité. Pour éviter ce travers, j'ai retenu les deux antinomies selon lesquelles se définissent habituellement les stratégies compositionnelles de la musique savante occidentale: la musique pure et la musique descriptive. À la première catégorie, j'associe l'intitulation autonyme et, à la seconde, l'intitulation référentielle.

Le titre autonyme me semble répondre à une conception italo-allemande de la composition musicale instaurée dès le XVII ${ }^{\mathrm{e}}$ siècle ${ }^{13}$. Selon cette représentation, l'expression musicale et le message exprimé ne font qu'un. C'est cette esthétique qu'a voulu défendre et théoriser Eduard Hanslick dans Vom Musikalisch-Schönen (1854) où il résume l'essence de ce formalisme allemand: «En musique, il n'y a pas de contenu opposable à la forme, parce qu'il n'y a pas de forme séparable du contenu " (1986: 163). C'est cette unicité de contenu et de forme qu'expriment les titres des œuvres orchestrales de Karlheinz Stockhausen (1928-2007): Formel, Spiel/Jeu, Punkte/ Points, Gruppen/Groupes, Carré, Mixtur/Mixture. Il en est de même de ces 4' 33" d'immobilité du pianiste devant son clavier écoutant les murmures de la salle, qui placent John Cage (1912-1992) au premier rang 
des compositeurs friands de titres désignant avant tout l'état de l'œuvre. Les titres donnés en anagrammes par Robert Schumann (1810-1856) à plusieurs de ses œuvres pianistiques pourraient laisser croire à un glissement de l'esthétique autonyme allemande vers la référence extérieure. Très certainement, il y a un peu de cela puisque ses Variations sur le nom d'ABBEG affichent le nom de famille de la jeune pianiste prénommée Meta dont il avait remarqué la beauté envoûtante. Mais il importe surtout d'observer qu'il n'y a en cette ouvre aucune prétention au portrait; tout au plus une dédicace et un jeu de mot tourné vers la musique en soi puisque, dans la nomenclature allemande, les lettres A-B-B-E-G désignent les notes la-si bémol-si bémol-mi-sol qui constituent le thème musical de l'œuvre. La musique se déroule dans le rapport d'un thème à ses variations. C'est d'abord ce que le titre schumannien nous annonce. Le procédé est similaire pour l'opus 8, Carnaval, soustitré Scènes mignonnes sur quatre notes. Il n'y a dans cette œuvre aucune agitation carnavalesque, ni évocation de masques. Comme pour les Variations, le titre permet de remonter à la source d'inspiration du motif musical. À la date de composition de l'œuvre, 1835, Schumann s'était fiancé secrètement à Ernestine von Fricken, originaire de la ville d'Asch en Bohème. Le compositeur s'est inspiré de l'épellation topographique pour composer son motif musical: As-C-H, soit la bémol-do-si, avec la variante à quatre notes, A-Es-C-H, soit la, mi bémol, do, si. Poursuivant le jeu des associations, le mot allemand Asche signifiant "cendre», Schumann en est arrivé au titre Carnaval, évocateur de ces réjouissances printanières déclenchées par l'Aschermittwoch, le mercredi des Cendres. Toute peinture de situation est absente de l'œuvre.

En revanche, l'expérience référentielle a la France pour terre d'origine. L'union de la musique et de la danse dans un même projet narratif et allégorique a été au départ de la symbolisation du musical. Forme stylisée par l'association initiale de la musique et de la danse, la suite s'est d'abord transformée en icône de leur valence signifiante. Puis, les compositeurs français se sont laissés entraîner dans une spirale de renvois symboliques qui a pris avec Couperin «Le Grand» la stature d'imposante majesté qu'on lui connaît depuis et dont la puissance de séduction semble n'avoir de cesse.

Mais, à la lumière de l'histoire des courants esthétiques, des nuances s'imposent dans le maniement de ces concepts. Ainsi, l'apparition d'un des pôles de l'antinomie intrinsèque/extrinsèque, pour être prévalente dans une culture, n'exclut point l'attrait vers le terme opposé. Les sociologues de la musique diraient avec raison que le désir de se faire reconnaitre sur le terrain de l'autre porte à utiliser son langage: cette mutabilité est le gage de la célébrité et de la prospérité que l'œuvre rapporte à son auteur. Pour cette raison, Telemann a beaucoup composé dans le goût français - Die Tagenzeiten / Les Heures $d u$ jour, en sont un exemple. La réception enflammée réservée aux Saisons de Vivaldi à Paris est tout à fait révélatrice. En contrepartie, Couperin non seulement aspire à réunir le goût des nations - Les Nations, Les Goûts réunis - mais, dans cette orgie de titres référentiels que sont les ordres, il se ménage de brefs moments de préoccupation formaliste: «La Sezile, Pièce croisée sur le grand clavier ", "Les Agrémens", pièce consacrée aux formules d'ornementation si fertiles dans la musique baroque, comme le pincé, le port de voix, le tremblement, l'arpègement, le coulé, la broderie, l'appogiature et le trille. Rameau aussi sacrifie au principe d'autonymie pour assurer son européanité en attirant, grâce aux titres, l'attention sur la pure forme musicale: «Les Trois mains» et la «Fanfarinette» du Deuxième Livre, «Les Triolets» et «L'Enharmonique» du Troisième Livre.

Les compositeurs de notre temps partagent aussi leur intérêt entre les deux sortes d'intitulés, tantôt renvoyant l'œuvre à elle-même, pour initier l'auditeur à sa structure, tantôt, au contraire, la dépliant vers l'extériorité. Tōru Takemitsu (1930-1996) jongle ici avec les deux manières que je laisse au lecteur le soin de départager: To the Edge of Dream (1983), Spirit Garden (1994), Lento in Due Movimenti (1950). Les œuvres de Sofia Gubaïdulina (1931) oscillent aussi 
entre les deux pôles: Allegro rustico (1963), The Garden of Joy and Sorrow (1980).

Quoi de plus normal que le Ravel des Jeux d'eau (1901) et le Debussy des Estampes (1903) se soient réclamés de la filiation de Couperin et de Rameau, l'impressionnisme musical étant avant tout un fait français, une célébration des noces de l'ouïe et de la vue. C'est dans ce courant que s'épanouit magnifiquement l'art des images de Couperin, à l'invocation du vocable, de la musique et de la danse, car Les Fées sont d'exquises danseuses ${ }^{14}$ et elles assurent ces mystérieuses, subtiles et signifiantes transitions vers La Valse ${ }^{15}$ et le Boléro ${ }^{16}$. Les miroirs de l'œuvre reflètent les références du syntagme littéraire qui la désigne, créant des intertextualités littéraires, picturales, voire musicales, données pour référents. Liszt (1811-1886) avait initié ces trois modes ouvrant par là d'insondables perspectives au titre référentiel dans ses Années de Pèlerinage: Sonetto 123 del Petraca, La Vallée d'Obermann ${ }^{17}$, Spozalizio ${ }^{18}$, Canzone ${ }^{19}$. Moussorgski inscrivit, dans la même veine, ses Tableaux d'une exposition (1874) inspirés des œuvres picturales de son ami architecte Victor Hartmann. La manière s'est étendue aux compositeurs du XXe siècle que ces jeux de ricochets engendrés par le Prélude à l'après-midi d'un faune (1894) de Debussy inspiré de Mallarmé ont littéralement subjugués.

Ce mouvement touche nos contemporains québécois qui en resserrent plus encore la poétique, conjuguant la représentation sonore à la représentation picturale. Jacques Hétu (1938) compose ses Images de la Révolution (1989) en s'inspirant des thématiques picturales de Jacques Louis David (17481825) dont il répercute les titres épiques: 1 . «Le Serment du Jeu de Paume», 2. «Le Convoi de La Bastille», 3. "Marat Assassiné», 4. "La Fête de l'Être suprême». John Rea (1944) ose davantage encore en combinant la référence picturale à la paraphrase sonore d'œuvres musicales préexistantes: Las Meninas (1990-91) reflète le Schumann des Kinderszenen mais aussi le Vélasquez de la petite Infante de Philippe IV entourée de ses demoiselles d'honneur et de ses nains typés ${ }^{20}$. Mais, en deçà de ces amplifications du procédé référentiel, l'essentiel de sa fonction réside dans cet acte fondamental de synesthésie qui consiste à poser la rame de portées à la place du canevas sur le chevalet et à lever les yeux au ciel pour entrevoir avec Messiaen les Couleurs de la Cité céleste.

\section{NOTES}

1. Le lecteur comprendra dès ici que cet article traite de l'œuvre musicale savante dans la tradition occidentale. Pour m'assurer de demeurer dans cette limite culturelle, je puiserai mes exemples chez les compositeurs répertoriés par François Verschaeve (2007).

2. J'emploie ce terme en pensant à la théorie de Saussure (1971) relative au langage parlé «articulé» autour de deux volets, le signifiant et le signifié. À l'opposé, la musique est considérée comme un langage "inarticulé» parce qu'elle est dépourvue de signifié.

3. Felix Mendelssohn rapporté par B.M. Teplov (1966: 10).

4. De ce compositeur, pensons à Till l'espiègle (1892) et à Ainsi parlait Zarathoustra (1896).

5. De ce compositeur, pensons aux «Fontaines » et aux «Pins », tirés du poème symphonique Rome (1917).

6. Pensons aux Planets (1914-1916) de Gustav Holst (1874-1934).

7. Pensons au poème symphonique d'Alexandre Borodine (18331887), Dans les steppes de l'Asie centrale (1880).

8. Il existe bien une suite à l'anglaise, qui est à toute fin pratique une extension du genre français; sa particularité réside essentiellement dans la liberté de déroger au principe de la tonalité uniforme. J.-S. Bach justifie par le respect ou la transgression de la règle de la tonalité unique ses appellations de Suite française et de Suite anglaise.

9. J'emprunte ces deux appellations successives, «titres-personnage" et «titres-caractère », à l'étude de Pierre Saby (2008) citée plus haut. Je m'en inspire pour désigner les «titres-paysage».

10. L'expression m'est inspirée de Diderot qui écrit: «C'est la chose même que le peintre montre: les expressions du musicien et du poète n'en sont que des hiéroglyphes" ([1751] 1987: 72).

11. Pierre Saby a constitué un corpus des œuvres pour clavecin de Dagincourt et de Dandrieu à qui il consacre son étude des titres caractéristiques citée plus haut.

12. Titre d'une suite en six mouvements de Maurice Ravel (1917): "Prélude ", "Fugue», «Forlane», "Rigaudon ", « Menuet», "Toccate ». 13. Le lecteur pourra reconstituer la trajectoire qui m'a conduit à retenir ces concepts en se rapportant à C. Dauphin (2001). Je démontre dans cet ouvrage comment le débat autour du postulat de l'imitation référentielle en France, dès le début du XVIII e siècle, a raffermi le projet d'une musique française essentiellement descriptive et, en même temps, favorisé l'émergence d'une théorie formaliste européenne revendiquant une esthétique de la musique tournée uniquement vers les propriétés immanentes de cet art.

14. Tiré du 2e Livre des Préludes de Debussy. 
15. Euvre de Maurice Ravel.

16. Euvre de Maurice Ravel.

17. Par ce titre, Liszt fait référence au roman épistolaire Oberman (1804) d'Étienne de Senancour (1770-1846) auquel il emprunte l'une des trois épigraphes de sa pièce: "Que veux-je? que suis-je? que demander à la nature? [...] Toute cause est invisible, toute fin trompeuse; toute forme change, toute durée s'épuise: [...] je sens, j'existe pour me consumer en désirs indomptables, pour m'abreuver de la séduction d'une (sic) monde fantastique, pour rester atterré de sa voluptueuse erreur " (Lettre 53; 1978: 32).

18. Par ce titre, Liszt fait référence au tableau de Raphaël ayant pour thème Le Mariage de la Vierge.

19. Par ce titre, Liszt fait référence au chant du gondolier "Nessùn maggior dolore" de l'Otello de Rossini.

20. Sur ces 21 Variations transformelles, voir l'article de V. Brauer, S. Lacasse et R. Villemaire (1996).

\section{RÉFÉRENCES BIBLIOGRAPHIQUES}

BraUer, V., S. LACASSE et R. Villemaire [1996]: "Analyse

d'une œuvre hypertextuelle, Las Meninas, vingt et une variations

transformelles sur les Kinderszenen de Robert Schumann, de John Rea ", Cahiers de l'ARMuQ, no 17, juin, 35-44.

CHABANON, M. P. G. [(1764 et 1779) 1969]: Observations sur la musique et principalement sur la métaphysique de l'art, Genève, Slatkine Reprints.
COUperin, F. [1980]: Euvres complètes: Pièces de clavecin, premier Livre, Monaco, L'Oiseau-Lyre/Les Remparts.

DAUPHIN, C. [2001]: La Musique au temps des encyclopédistes, Ferney-

Voltaire, CIEDS.

DideroT, D. [(1751) 1987] : Lettres sur les sourds et les muets, dans Écrits sur la musique, Paris, Lattès, 69-74.

ESCAL, F. [1996] : Aléas de l'œuvre musicale, Paris, Hermann.

HANSLICK, E. [(1854) 1986]: Du beau dans la musique, Paris, Bourgois. INGARTEN, R. [1989]: Qu'est-ce qu'une œuvre musicale?, Paris, Bourgois. LISZT, F. [1978] : Années de Pèlerinage: première année, Suisse, Munich, G. Henle.

MALlarmÉ, S. [1945]: «L'Après-midi d'un faune», dans Euvres complètes, Paris, Bibliothèque de la Pléiade, 50-53

PAulus, J. [1972]: La Fonction symbolique et le langage, Bruxelles, C.

Dessart.

Pseudo-Longin [1995]: Traité du Sublime, Paris, Librairie Générale Française.

SABY, P. [2008]: "Titres et caractérisation dans la musique instrumentale française : imitation, suggestion et illusion ", Actes $d u$ colloque Autour de Watteau (30 novembre et $1^{\text {er }}$ décembre 2006), Presses Universitaires de Valenciennes (édition numérique), en instance de parution.

SAUSSURE, F. de [1971]: Cours de linguistique générale, Paris, Payot. StraVINSKY, I. [(1962) 2000]: Chroniques de ma vie, Paris, Denoël. TePlov, B. M. [1966]: Psychologie des aptitudes musicales, Paris, PUF. VerschaeVe, F. [2007]: What's What in Titles of Classical Music... and beyond. A Dictionary of Titles, Waterdown (Ontario), F. Verschaeve. 